\title{
Retraction Note: Note on "Smaller Explicit Superconcentrators" by N. Alon and M. Capalbo
}

\author{
L. A. Bassalygo \\ Kharkevich Institute for Information Transmission Problems, \\ Russian Academy of Sciences, Moscow, Russia \\ e-mail: bass@iitp.ru
}

Received October 29, 2020; revised October 29, 2020; accepted October 29, 2020

DOI: $10.1134 / \mathrm{S} 0032946020040092$

The author has retracted this article [1], since a critical error was found in the proof of the main result after its publication, thus making it invalid. The article [1] was written as a note to a paper by N. Alon and M. Capalbo [2].

The author is grateful to Professor A. Yavuz Oruç (Department of Electrical and Computer Engineering, University of Maryland, College Park, MD) for pointing to the fallacy in the original article.

\section{REFERENCES}

1. Bassalygo, L.A., Note on "Smaller Explicit Superconcentrators" by N. Alon and M. Capalbo, Probl. Peredachi Inf., 2019, vol. 55, no. 3, pp. 106-108. https://doi.org/10.1134/S0032946019030086

2. Alon, N. and Capalbo, M., Smaller Explicit Superconcentrators, Internet Math., 2004, vol. 1, no. 2, pp. 151-163. https://projecteuclid.org/euclid.im/1089229505 